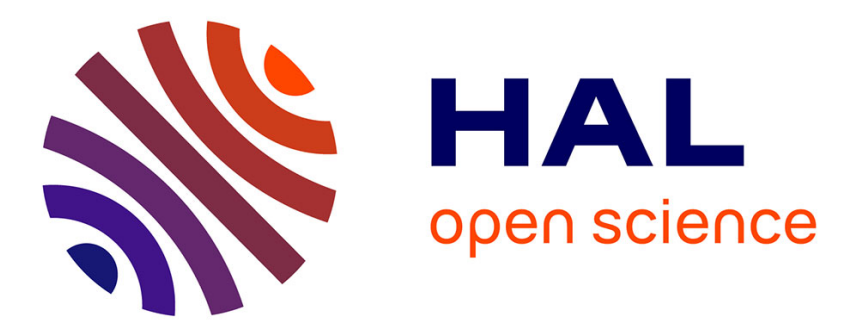

\title{
Microfluidic channel with embedded monolayer nanofibers for cell culture and co-culture
}

Boxin Huang, Yong He, Li Wang, Jian Shi, Jie Hu, Elrade Rofaani, Ayako Yamada, Yong Chen

\section{To cite this version:}

Boxin Huang, Yong He, Li Wang, Jian Shi, Jie Hu, et al.. Microfluidic channel with embedded monolayer nanofibers for cell culture and co-culture. Microelectronic Engineering, 2020, 225, pp.111235. 10.1016/j.mee.2020.111235 . hal-02565348v2

\section{HAL Id: hal-02565348 \\ https://hal.sorbonne-universite.fr/hal-02565348v2}

Submitted on 10 Dec 2020

HAL is a multi-disciplinary open access archive for the deposit and dissemination of scientific research documents, whether they are published or not. The documents may come from teaching and research institutions in France or abroad, or from public or private research centers.
L'archive ouverte pluridisciplinaire HAL, est destinée au dépôt et à la diffusion de documents scientifiques de niveau recherche, publiés ou non, émanant des établissements d'enseignement et de recherche français ou étrangers, des laboratoires publics ou privés. 


\title{
Microfluidic channel with embedded monolayer nanofibers for cell culture and co-culture
}

\author{
Boxin Huang ${ }^{\mathrm{a}}$, Yong He ${ }^{\mathrm{a}}$, Li Wang ${ }^{\mathrm{b}}$, Jian $\mathrm{Shi}^{\mathrm{b}}$, Jie Hu${ }^{\mathrm{a}}$, \\ Elrade Rofaani ${ }^{\mathrm{a}}$, Ayako Yamada ${ }^{\mathrm{a}}$ and Yong Chen ${ }^{\mathrm{a}}$ \\ ${ }^{a}$ PASTEUR, Département de chimie, École normale supérieure, PSL University, Sorbonne \\ Université, CNRS, 75005 Paris, France \\ ${ }^{\mathrm{b}}$ MesoBioTech, 231 Rue Saint-Honoré, 75001 Paris, France
}

\begin{abstract}
Mimicking the cellular microenvironment is important for organoids and organ on-a-chip studies. One of the current issues is to introduce vessel-like structures into the culture system to improve the cellular and tissue functions, which deserves particular efforts in design and systematic consideration. Based on a standard device configuration, we fabricated a vessel-like component which can be easily integrated for cell co-culture. This component consists of an embedded monolayer of gelatin nanofibers on the top of an open channel. It can then be enclosed with an upper plastic plate with molded chamber, channels and standard Luer connectors. Human umbilical vein endothelial cells (HUVECs) were firstly introduced into the vessel-like channel and cultivated threedimensionally with the help of a rotational device. Then, a flow was applied for cytoskeleton remolding, resulted in a dense and aligned HUVECs layer. Afterward, human glioblastoma cells (U87) were introduced in the upside of the fiber layer and a flow was also applied for the upper cell layer culture. Our results show adjunct formation of HUVEC and U87 cell layers on both sides of the monolayer of gelatin nanofibers, thereby providing a reliable support for a variety of co-culture assays.
\end{abstract}

Keywords: Microfluidics, Organ on-a-chip, Basement membrane, Blood vessel 


\section{Introduction}

Blood vessels, which transport blood cells, nutrients, and oxygen to the tissues and organs, are omnipresent in the body. Any abnormal function of the vessels may cause diseases and, thus, it is important to develop new vessel models taking into account physiological and pathological specification. In the current context of organ on-a-chip development, introducing artificial vessels become a key step toward higher body functions. In this regard, one has to create hollow tubular structures covered by endothelial cells ${ }^{1-3}$ and to co-culture other types of cells around the model vessels $^{4-6}$. By using different microfabrication techniques, various tubular structures have been proposed but plastic or elastomeric membranes were often used for co-culture, which differ significantly from the in vivo characteristics. In fact, the organ constituents such as alveolar and blood-brain-barrier are structurally formed with the help of basement membrane (BM), which is about $100 \mathrm{~nm}$ thick and made of specific extracellular matrix proteins. The challenge is then to mimic such a membrane and to make it integrative in a microfluidic device.

Previously, we have devised a so-called "patch method" by forming a monolayer of cross-linked gelatin nanofibers on a thin honeycomb frame $\mathrm{e}^{7-9}$. Although this device has been initially developed for pluripotent stem cell differentiation, the fabricated device can also be used for co-culture. Here, we developed an alternative approach to embed a suspended monolayer of cross-linked nanofibers in a microfluidic channel for co-culture under dynamic flow conditions. To make this method flexible and versatile, we adopted a standardized chip configuration in which the device is composed of two plates. A microchannel was defined in the lower plate and the monolayer nanofibers were embedded on top of the channel. The upper plate with elastomeric interlayer and Luer connectors was clamped above the lower plate with a mechanical device for a tight and reversible sealing. Finally, the device was connected to a fluidic controller for endothelial cell culture and co-culture under flow conditions. With this system, we achieved the formation of continuous endothelial cell layer covering the whole microchannel wall and the embedded nanofiber layer. Co-culture of HUVECs and U87 cells separated by porous monolayer of gelatin nanofibers was also demonstrated and the shear flow-induced cytoskeleton remolding has been revealed. Overall, this approach is successful and valuable for further investigation. 


\section{Materials and methods}

Reagents were purchased from Sigma-Aldrich (France) unless otherwise specified.

\subsection{Fabrication of the device}

We firstly fabricated the lower plate of the device (Fig. 1a). A $500 \mu \mathrm{m}$-wide, $4 \mathrm{~cm}$-long rectangular channel with $4 \mathrm{~mm}$-diameter reservoirs at its extremities was created in a $500 \mu \mathrm{m}$-thick layer of polydimethylsiloxane (PDMS, RTV 615, Eleco-EFD, France) by cutting plotter (Graphtec CE6000-40, France). After plasma treatment, the PDMS layer was bonded to a $1 \mathrm{~mm}$-thick glass slide. Afterward, a thin layer of non-reticulated viscous PDMS (v-PDMS) was deposited on top of the PDMS layer by microcontact printing. Here, the v-PDMS was firstly prepared by mixing the PDMS pre-polymer and the crosslinker at the ratio of 10:1 (w/w), degassed, and spin-coated on a glass substrate. Then, the PDMS/glass assembly was gently pressed against the v-PDMS. After separation, a monolayer of gelatin nanofibers was electrospun on the v-PDMS-coated surface, followed by a thermal treatment at $100^{\circ} \mathrm{C}$ for $10 \mathrm{~min}$. Thus, the gelatin nanofibers could be embedded in the PDMS, resulting in a free-standing monolayer of nanofibers. $10 \mathrm{wt} \%$ gelatin powder from porcine skin was dissolved in the mixed solution of acetic acid, ethyl acetate and DI water at the volume ratio of 21:14:10 prior to electrospinning. The gelatin solution was electrospun for $12 \mathrm{~min}$ from a syringe needle to the lower plate, which was placed on an aluminum foil at $10 \mathrm{~cm}$ distance from the needle tip. The solution was injected at $0.2 \mathrm{~mL} / \mathrm{h}$ by syringe pump, while the voltage between the needle and the foil was kept at $11 \mathrm{kV}$. Nanofibers in the reservoir areas were carefully removed by a fine cotton stick wet with DI water and the remaining nanofibers were treated with $0.2 \mathrm{M} \mathrm{N}$-(3-dimethylaminopropyl)-N'-ethylcarbodiimide hydrochloride and $0.2 \mathrm{M} \mathrm{N}$ hydroxysuccinimide in ethanol for chemical crosslinking for $4 \mathrm{~h}$ at room temperature (RT). As a result, a robust and flat monolayer of cross-linked gelatin nanofibers was formed, being suspended on top of the microfluidic channel.

An upper plate with two inlets (Fig. 1b left) or four inlets with a chamber (Fig. 1e) provided by Mesobiotech, ltd, was assembled with the lower plate to finalize the microfluidic device for the formation of endothelial cell layer (Fig. 1d) or co-culture of HUVECs and U87 (Fig. 1e), 
respectively. The upper and the lower plates can be reversibly assembled with a hand-screw clamp device and the assembly could be easily mounted on a rotation system for 3D culture (Fig. 1c). Both the clamp device and the rotation system were also provided by Mesobiotech, ltd.

\subsection{Characterization of the device}

The microchannel with embedded gelatin nanofibers of the lower plate were observed by scanning electron microscope (SEM, Hitachi S800) operated at $10 \mathrm{kV}$. To observe the cross-section of the channel, the lower plate was cut perpendicularly to the channel by a glass cutter and a blade. A $5 \mathrm{~nm}$-thick gold layer was sputtered on the samples prior to the SEM observation.

\subsection{Cell culture}

Human umbilical vein endothelial cells (HUVECs) and human glioblastoma cells (U87) purchased form Sigma-Aldrich, were cultured with endothelial cell growth medium (Cell Applications, Inc.) supplemented with 1\% (v/v) penicillin streptomycin (Pen Strep, Thermo Fisher Scientific) (Medium 1) and Dulbecco's modified Eagle medium (DMEM) supplemented with 10\% (v/v) fetal bovine serum and 1\% (v/v) Pen Strep (Medium 2, all reagents from Thermo Fisher Scientific), respectively, at $37{ }^{\circ} \mathrm{C}$ with $5 \% \mathrm{CO}_{2}$ in a humidified incubator.

After sterilization, the microfluidic channel was assembled with a two-inlet cover plate and reinforced by the clamp device. To promote cell adhesion, the channel was treated with $100 \mu \mathrm{g} / \mathrm{mL}$ poly-D-lysine (PDL) in Dulbecco's phosphate buffered saline (DPBS) at $4{ }^{\circ} \mathrm{C}$ overnight and $1 \%(\mathrm{v} / \mathrm{v})$ Geltrex hESC-Qualified matrix in DPBS at $37^{\circ} \mathrm{C}$ for $1 \mathrm{~h}$. HUVECs at the density of $1.5 \times 10^{7} / \mathrm{mL}$ in $50 \mu \mathrm{L}$ Medium 1 were seeded into the channel and incubated at $37{ }^{\circ} \mathrm{C}$ with $5 \% \mathrm{CO}_{2}$ for $4 \mathrm{~h}$ (Fig. $1 \mathrm{~d}$ ). During the first hour of incubation, the device was rotated $90^{\circ}$ three times to allow cell attachment on the entire channel surface, including the exposed area of the nanofiber monolayer. $200 \mu \mathrm{L}$ of Medium 1 was added into the channel and the device was incubated overnight in the $\mathrm{CO}_{2}$ incubator. HUVECs were then cultured under a dynamic condition with a flow of the culture medium at 0.1 $\mathrm{mL} / \mathrm{h}$, controlled by a syringe pump, for 2 days.

For co-culture, the two-inlet upper plate was replaced by a four-inlet one equipped with a microfluidic channel and a central culture chamber (Fig. 1c, 1e). 5 x 106 U87 cells suspended in 50 
$\mu \mathrm{L}$ of Medium 2 supplemented with $10 \%$ (v/v) Geltrex were seeded on the other side of the nanofiber monolayer through the inlet connected to the upper channel. After $2 \mathrm{~h}$ incubation at $37{ }^{\circ} \mathrm{C}$ with 5\% $\mathrm{CO}_{2}$, Medium 1 and Medium 2 were injected to fill up the lower channel and upper culture chamber, respectively. Both channels were perfused with a constant flow for further culture.

\subsection{Control of microflow condition}

After cell adhesion, a constant flow of the culture medium at the rate of $0.1 \mathrm{~mL} / \mathrm{h}$ was applied using a syringe pump. The co-culture was also performed with the same flow rate but with different culture media above and below the nanofiber-supported cell layers. The shear stress $(\tau)$ in the channel is given by

$$
\tau=\frac{6 \mu \mathrm{Q}}{\mathrm{wh}^{2}}
$$

where $\mu$ is the dynamic viscosity of the medium $\left(0.012\right.$ dyn.s $\left./ \mathrm{cm}^{2}\right), Q$ the flow rate $(0.1 \mathrm{~mL} / \mathrm{h}), \mathrm{w}$ and $\mathrm{h}$ the width $(500 \mu \mathrm{m})$ and the height $(500 \mu \mathrm{m})$ of the channel, respectively. $\tau$ in our condition is $0.02 \mathrm{dyn} / \mathrm{cm}^{2}$.

\subsection{Immunofluorescence imaging}

HUVECs were fixed with 4\% (w/v) paraformaldehyde in DPBS for $15 \mathrm{~min}$ at RT and rinsed with DPBS three times, prior to the incubation with $0.5 \%(\mathrm{w} / \mathrm{v})$ Triton X-100 in DPBS for 10 min at RT for permeabilization. After rinsing with DPBS three times, the cells were further incubated with $3 \%(\mathrm{w} / \mathrm{v})$ bovine serum albumin, $0.1 \%(\mathrm{w} / \mathrm{v})$ Triton X-100, and $0.1 \%(\mathrm{w} / \mathrm{v})$ sodium azide in DPBS for $30 \mathrm{~min}$ at room temperature. Subsequently, the cells were incubated with Atto 488 Phalloidin and DAPI (Thermo Fisher Scientific) in DPBS for $20 \mathrm{~min}$ at RT to visualize actin filaments and nuclei, respectively. After three times rinsing with DPBS, cell morphologies and distribution were observed by confocal microscope (LSM 710, Zeiss).

\subsection{Cell labeling}

Cell Tracker ${ }^{\mathrm{TM}}$ fluorescent probes (Thermo Fisher Scientific) were used to distinguish the two cell types. $1 \mu \mathrm{M}$ Cell Tracker ${ }^{\mathrm{TM}}$ Deep Red (C34565) and $25 \mu \mathrm{M}$ Cell Tracker ${ }^{\mathrm{TM}}$ Green (2925) in serum free medium were used to label HUVECs and U87 cells, respectively. Prior to labeling, both 
cells were cultured separately in tissue culture dishes (Fisher Scientific) with the appropriate medium. After medium removal, pre-warmed Cell Tracker ${ }^{\mathrm{TM}}$ solutions were gently added to the corresponding dishes and the cells were incubated with the solutions for $30 \mathrm{~min}$ at $37^{\circ} \mathrm{C}$. Then, the cells were trypsinized by TrypLE Express (Thermo Fisher Scientific), collected by centrifugation, and seeded into the device as described above. After $24 \mathrm{~h}$ of co-culture, the cells were imaged by fluorescence microscope (Observer Z1, Zeiss).

\section{Results and discussion}

The gelatin nanofibers embedded in the top PDMS layer can be easily observed bySEM (Fig. 2). In Fig. 2a, porous structures of the fiber layer can be clearly seen, indicating that all pores are small enough to capture all the cells during cell seeding. Although the exact thickness of the nanofibers layer cannot be determined from Fig. $2 b$ and $2 c$, the high porosity observed in Fig. 2a suggests the formation of a monolayer of nanofibers and a thickness of few hundred nanometers. Such a monolayer of crosslinked nanofibers has many advantages for cell culture and co-culture in terms of nutrient diffusion, cell to cell interaction across the monolayer, and the structure closely mimicking the basement membrane. As seen in Fig. 2a-c, the monolayer is flat across the microchannel without deformation, proving that it is well integrated and supported by the thin PDMS layer on the surface of the lower plate.

Both the channel and the nanofiber embedding layers are in PDMS. Once the lower plate is clamped with the upper plate, a conformal contact is achieved to prevent leakage. Other methods such as PDMS casting can also be used to produce the lower plate structure. This device configuration might be versatile for many applications involving cell-cell, cell-organoid and organorgan co-culture.

After incubation for $24 \mathrm{~h}$, the cell layers got confluent (Fig. 3a). The monolayer of endothelial cells with luminal structure created in the microchannel is similar to the small blood vessels in vivo (Fig. 3b) except the square channel shape. The fabrication of a rounded channel is possible with more sophisticated patterning techniques. The cell morphology can be more clearly seen in Fig.3c from which the cell number can also be deduced by counting the nuclei of the cells with DAPI 
marker. Remarkably, we observed a higher cell density on the top wall than the bottom one (Fig.3d), suggesting the suitability of the suspended monolayer nanofibers for endothelial cell culture.

It is known that endothelial cells in blood vessels are exposed to hemodynamic forces associated with blood flow, which cause shear stress and friction of blood with the luminal cell surface. ${ }^{10-12}$ In our case, we observed remarkable changes of the HUVECs morphology on top and bottom walls of the channel after incubation for $48 \mathrm{~h}$ under dynamic flow conditions and cells were clearly aligned along the flow direction (Fig. 4). Under static conditions, cells were not aligned on both layers after incubation for 3 days. In addition, some cells at the bottom became round and tended to detach from the substrate, which means that the shear stress induced under dynamic culture conditions is necessary for long-term maintenance of HUVECs culture.

U87 cancer cells were chosen to demonstrate co-culture with HUVECs with suspended gelatin nanofibers above the channel. We seeded U87 directly on to the backside of the HUVEC layer separated by the monolayer of gelatin nanofibers. After incubation for $24 \mathrm{~h}$, HUVECs and U87 cells were labeled with red and green markers, respectively. As can be seen in Fig. 5, U87 cells adhered well on the gelatin nanofibers and HUVECs remained healthy during co-culture. Here, the gelatin nanofibers served as a mechanic support for cell adhesion and cell layer formation. Such a device configuration should be useful for co-culture of other cells and more systematic studies are needed to understand more clearly how cells interact each other across a monolayer of nanofibers. More interestingly, tumor angiogenesis and tumor metastasis can be studied using such a device ${ }^{13-17}$. In such a case, it should be possible to form tumor spheroids close to a vessel and to observe the interplay of the two systems. More generally, our approach can be applied to generate different types of tissues and different organ constituents involving artificial vessels and/or artificial basement membranes.

\section{Conclusion}

We have developed a microfluidic device with an embedded monolayer of nanofibers in order to achieve a generalized device configuration for co-culture-based assays. This device has firstly been used for three-dimensional culture of endothelial cells (HUVECs) on the microfluidic channel 
wall and the fiber monolayer. We also examined the morphology changes of HUVECs as a function of shear stress. With the same device, co-culture of HUVECs and U87 cells has been achieved, showing a continuous layer of HUVECs on the downside and a U87 cell layer on the upside of the fiber layer, thereby proving the potential of this new approach with an artificial yet physiologically relevant basement membrane for a plethora of possible applications. Finally, the device presented in this work will soon be commercially available.

\section{Acknowledgements}

This work was supported by Agence de Recherche Nationale under contract ANR-17-CE09-0017 (AlveolusMimics), European Commission Cost Action BIONECA (CA 16122), DIM ELICIT program of Ile-de-France, and PSL Valorization through Pre-maturation project. Boxin Huang also thanks to the Oversea Study Program of Guangzhou Elite Project. 


\section{References}

[1] N. Koike, D. Fukumura, O. Gralla, P. Au, J.S. Schechner, R.K. Jain, Creation of long-lasting blood vessels, Nature 428(6979) (2004) 138-139.

[2] K. Chwalek, M.V. Tsurkan, U. Freudenberg, C. Werner, Glycosaminoglycan-based hydrogels to modulate heterocellular communication in in vitro angiogenesis models, Sci Rep 4 (2014) 4414.

[3] S.M. Ehsan, K.M. Welch-Reardon, M.L. Waterman, C.C. Hughes, S.C. George, A three-dimensional in vitro model of tumor cell intravasation, Integr Biol (Camb) 6(6) (2014) 603-10.

[4] S.N. Bhatia, D.E. Ingber, Microfluidic organs-on-chips, Nat Biotechnol 32 (2014) 760.

[5] H. Cho, J.H. Seo, K.H.K. Wong, Y. Terasaki, J. Park, K. Bong, K. Arai, E.H. Lo, D. Irimia, ThreeDimensional Blood-Brain Barrier Model for in vitro Studies of Neurovascular Pathology, Scientific reports 5 (2015) 15222-15222.

[6] L.L. Bischel, K.E. Sung, J.A. Jimenez-Torres, B. Mader, P.J. Keely, D.J. Beebe, The importance of being a lumen, FASEB J 28(11) (2014) 4583-90.

[7] Y. Tang, F.P. Ulloa Severino, F. Iseppon, V. Torre, Y. Chen, Patch method for culture of primary hippocampal neurons, Microelectronic Engineering 175 (2017) 61-66.

[8] Y. Tang, L. Liu, J. Li, L. Yu, L. Wang, J. Shi, Y. Chen, Induction and differentiation of human induced pluripotent stem cells into functional cardiomyocytes on a compartmented monolayer of gelatin nanofibers, Nanoscale 8(30) (2016) 14530-40.

[9] Y. Tang, L. Liu, J. Li, L. Yu, F.P.U. Severino, L. Wang, J. Shi, X. Tu, V. Torre, Y. Chen, Effective motor neuron differentiation of hiPSCs on a patch made of crosslinked monolayer gelatin nanofibers, Journal of Materials Chemistry B 4(19) (2016) 3305-3312.

[10] M. Raasch, K. Rennert, T. Jahn, S. Peters, T. Henkel, O. Huber, I. Schulz, H. Becker, S. Lorkowski, H. Funke, A. Mosig, Microfluidically supported biochip design for culture of endothelial cell layers with improved perfusion conditions, Biofabrication 7(1) (2015) 015013.

[11] N.R. Wevers, D.G. Kasi, T. Gray, K.J. Wilschut, B. Smith, R. van Vught, F. Shimizu, Y. Sano, T. Kanda, G. Marsh, S.J. Trietsch, P. Vulto, H.L. Lanz, B. Obermeier, A perfused human blood-brain barrier on-a-chip for high-throughput assessment of barrier function and antibody transport, Fluids Barriers CNS 15(1) (2018) 23. 
[12] P.P. Partyka, G.A. Godsey, J.R. Galie, M.C. Kosciuk, N.K. Acharya, R.G. Nagele, P.A. Galie, Mechanical stress regulates transport in a compliant 3D model of the blood-brain barrier, Biomaterials 115 (2017) 30-39.

[13] T. Magdeldin, V. Lopez-Davila, J. Pape, G.W. Cameron, M. Emberton, M. Loizidou, U. Cheema, Engineering a vascularised 3D in vitro model of cancer progression, Sci Rep 7 (2017) 44045.

[14] H.-F. Tsai, A. Trubelja, A.Q. Shen, G. Bao, Tumour-on-a-chip: microfluidic models of tumour morphology, growth and microenvironment, Journal of the Royal Society, Interface 14(131) (2017) 20170137.

[15] M. Chung, J. Ahn, K. Son, S. Kim, N.L. Jeon, Biomimetic Model of Tumor Microenvironment on Microfluidic Platform, Adv Healthc Mater 6(15) (2017).

[16] A. Sobrino, D.T. Phan, R. Datta, X. Wang, S.J. Hachey, M. Romero-Lopez, E. Gratton, A.P. Lee, S.C. George, C.C. Hughes, 3D microtumors in vitro supported by perfused vascular networks, Sci Rep 6 (2016) 31589.

[17] N.G. Avci, Y. Fan, A. Dragomir, Y.M. Akay, M. Akay, Investigating the Influence of HUVECs in the Formation of Glioblastoma Spheroids in High-Throughput Three-Dimensional Microwells, IEEE Trans Nanobioscience 14(7) (2015) 790-6. 


\section{Figure caption}

Figure 1. Schematic of the device fabrication process and the cell culture and co-culture configuration:

(a) Lower plate, (b) Device assembly, (c) Rotational culture device, (d) and (e) Side and cross-section views of culture and co-culture configurations, respectively.

Figure 2. Scanning electron microscopy images of a microfluidic channel with embedded monolayer nanofibers. (a) Top view, (b) Cross-section view and (c) Enlarge cross-section view.

Figure 3. Confocal images of HUVECs cultured in microfluidic channel for $24 \mathrm{~h}$. 3D views (a) and cross-sectional views (b) of the HUVECs on all the surfaces. The morphology (c) and cell number (d) of HUVECs on gelatin nanofibers and at the bottom of channel are presented, respectively. Scale bars, $300 \mu \mathrm{m}$.

Figure 4. Phase contrast images of HUVECs on the top (below the fiber layer) and bottom wall of the channel at day 1 and 3. Scale bars, $100 \mu \mathrm{m}$.

Figure 5. Fluorescence images of U87 cells above the fiber layer, HUVECs below the fiber layer and HUVECs on the bottom wall. Staining: Cell Tracker ${ }^{\mathrm{TM}}$. Scale bars, $100 \mu \mathrm{m}$. 
Figure.1.

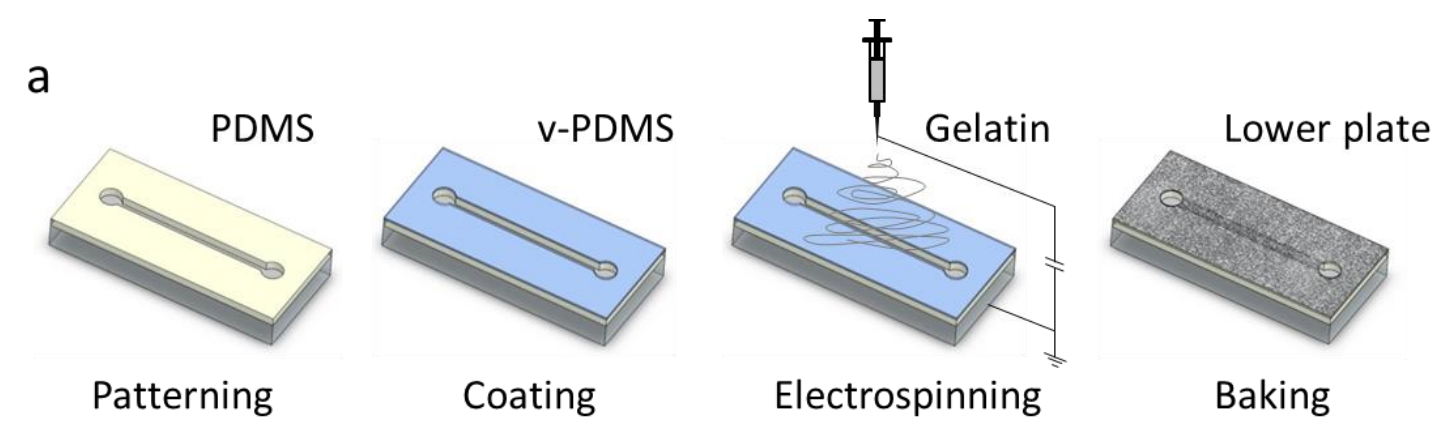

b

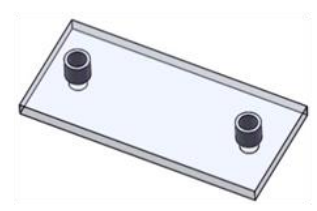

Upper plate

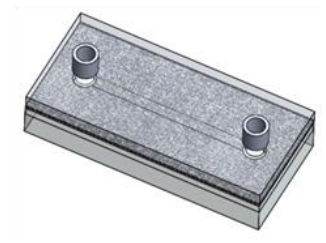

C

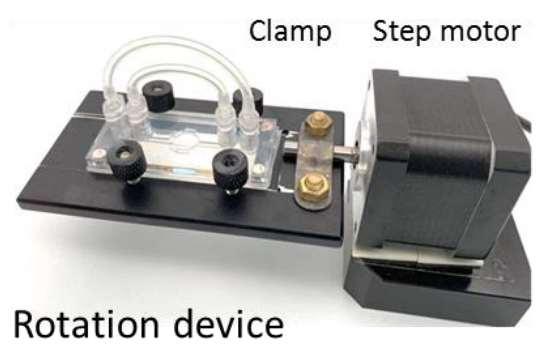

d

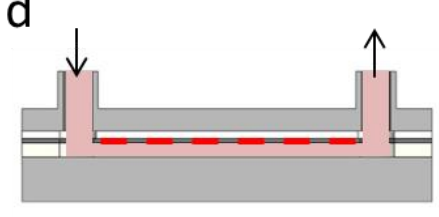

Cell Culture

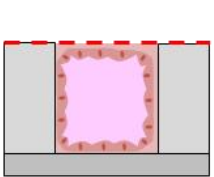

Co-culture

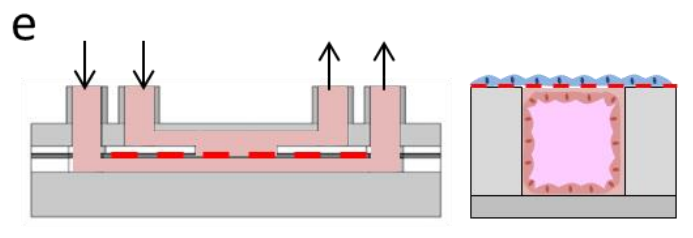

Figure.2
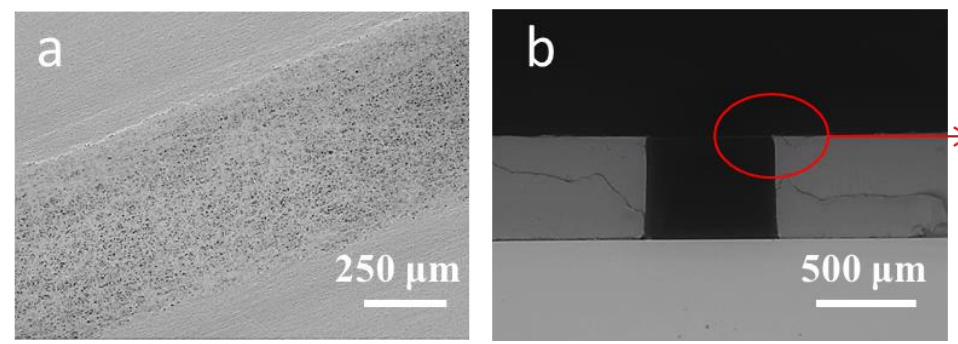
Figure.3.
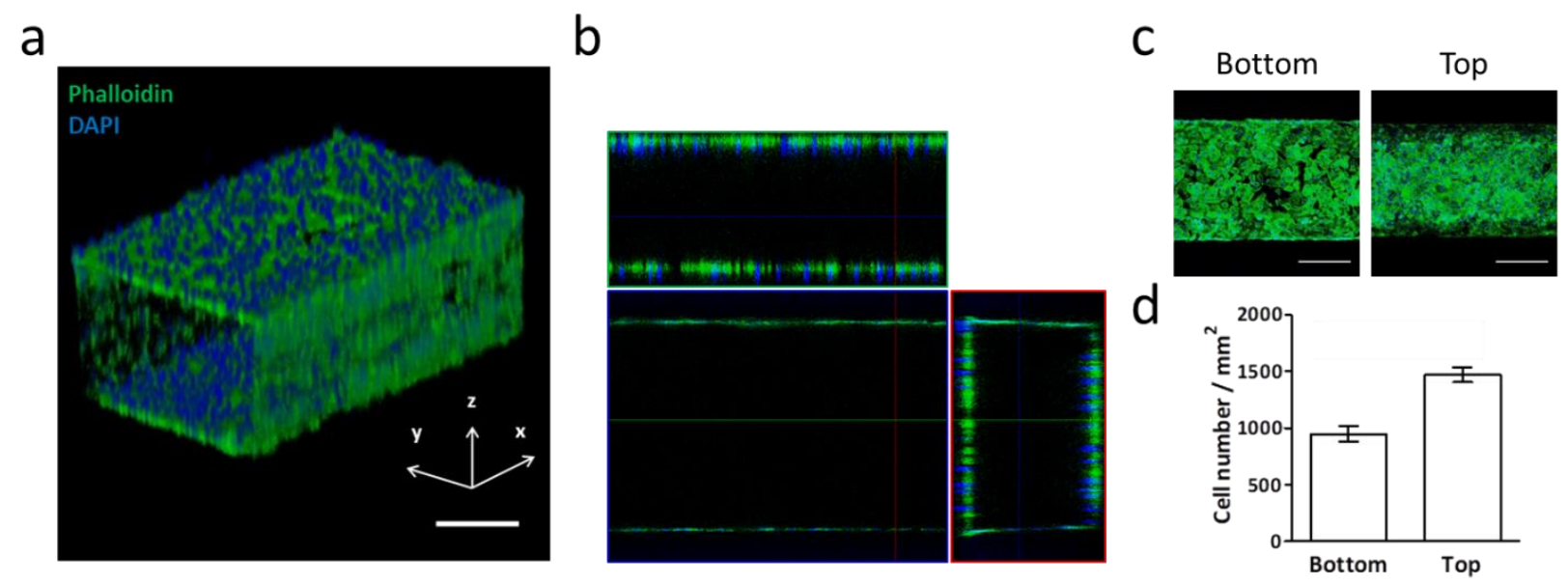

Figure.4.

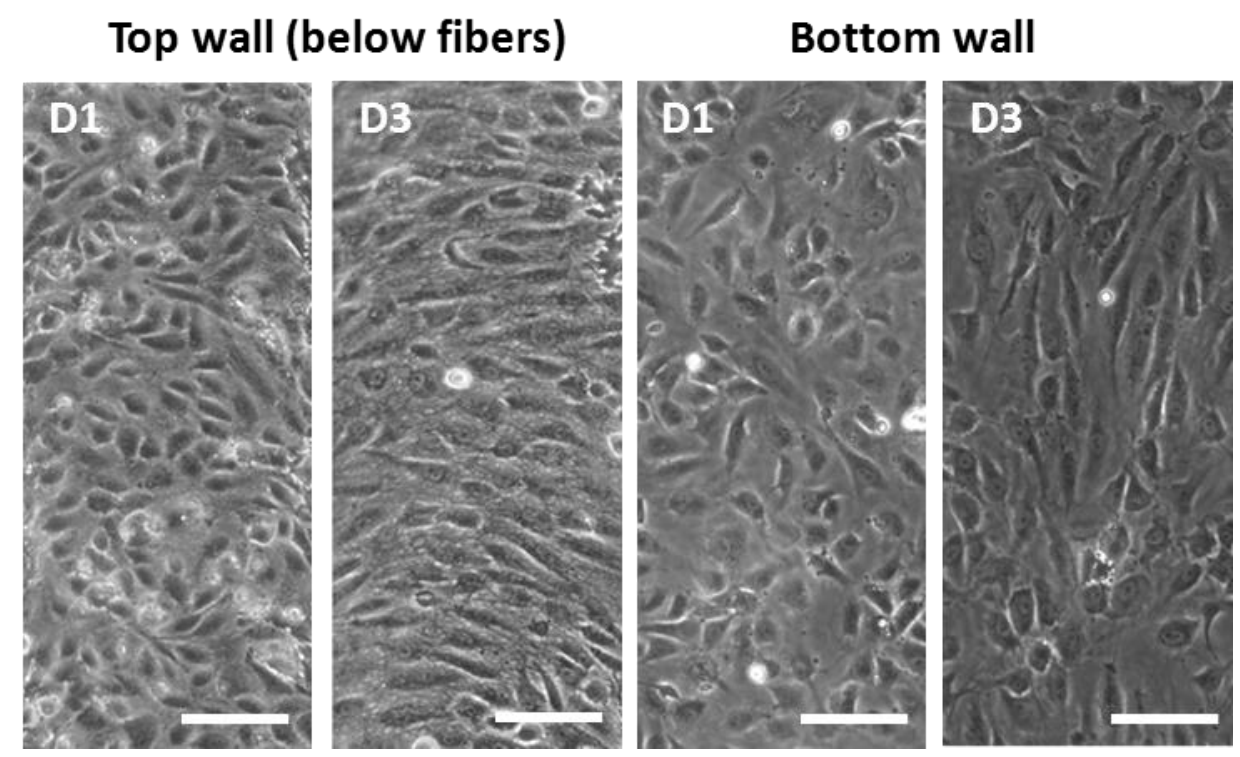


Figure.5.

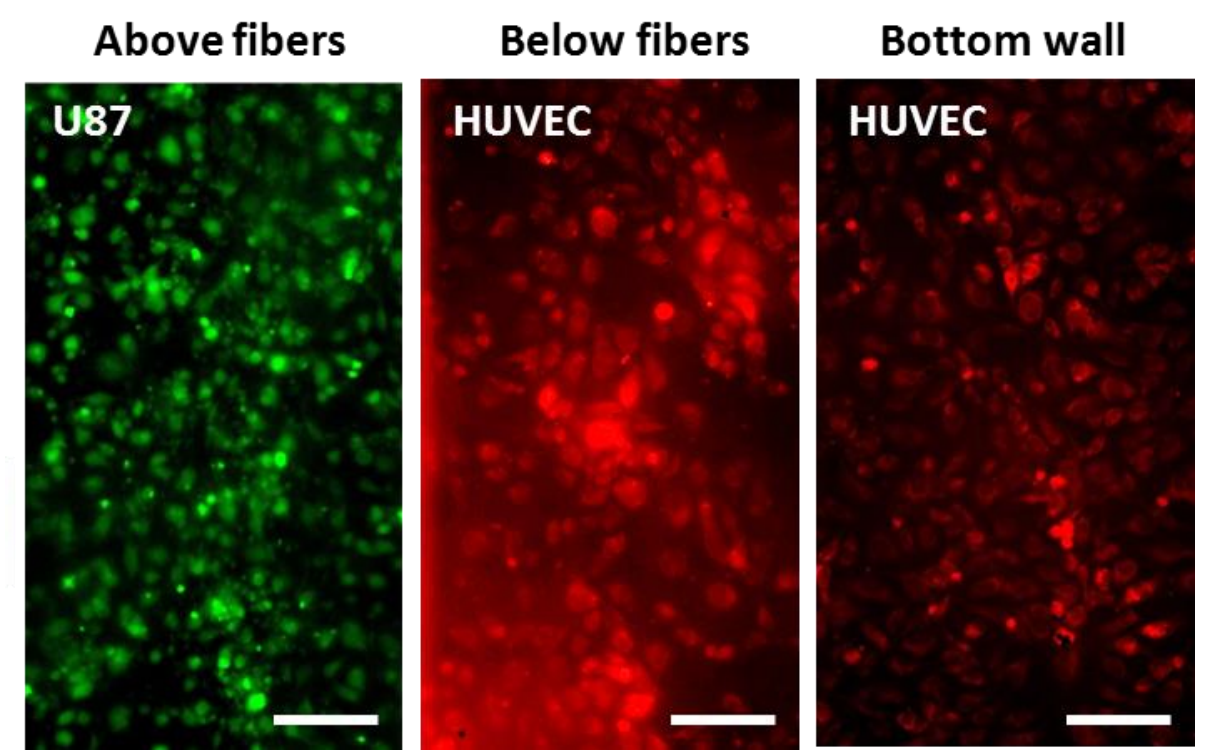

